\title{
Repercussões da pandemia da Covid-19 no cuidado de lactentes nascidos prematuros
}

\author{
Repercussions of the Covid-19 pandemic in the care of premature infants \\ Repercusiones de la pandemia de Covid-19 en el cuidado de lactantes prematuros
}

Altamira Pereira da Silva Reichert ${ }^{1}$ (B) Anna Tereza Alves Guedes ${ }^{1}$ (D) Anniely Rodrigues Soares ${ }^{1}$ (1) Paloma Karen Holanda Brito ${ }^{2}$ (1) Iolanda Carlli da Silva Bezerra ${ }^{1}$ (1)

Layla Caroline Lino da Silva ${ }^{3}$ (1)

Tayanne Kiev Carvalho Dias ${ }^{1}$ (i) Nathanielly Cristina Carvalho de Brito Santos $^{3}$ (b)

1. Universidade Federal da Paraíba. João Pessoa, PB, Brasil.

2. Universidade Federal de Campina Grande, Graduação em Enfermagem. Cajazeiras, PB, Brasil.

3. Universidade Federal de Campina Grande. Cuité, PB, Brasil.
Autor correspondente:

Anna Tereza Alves Guedes.

E-mail: annaterezag@gmail.com.

Recebido em 04/05/2021.

Aprovado em 21/07/2021.

DOI:https://doi.org/10.1590/2177-9465-EAN-2021-0179

\section{Resumo}

Objetivo: O estudo objetivou compreender as repercussões da pandemia da Covid-19 no cuidado de lactentes prematuros, na perspectiva de mães e profissionais de saúde. Método: Foram realizadas entrevistas semiestruturadas nos meses de junho e julho de 2020, por meio de ligação telefônica, com 14 mães e quatro profissionais de saúde do serviço de follow-up de uma maternidade pública da Paraíba, Brasil. Resultados: A partir da análise temática indutiva, os impactos da pandemia no cuidado ao lactente nascido prematuro, foram: sobrecarga e afastamento dos profissionais dos serviços de saúde, desativação temporária da unidade mãe canguru, descontinuidade da assistência ao prematuro, medo materno de expor a criança à Covid-19 e baixa condição socioeconômica. Foram elencadas estratégias de enfrentamento para o cuidado dos lactentes durante a pandemia como: maior espaçamento das consultas, acompanhamento por meio telefônico e cumprimento das medidas de biossegurança. Conclusão e implicações para a prática: A pandemia exigiu adaptações na assistência, tornando necessárias novas formas de cuidado a essas crianças, como exemplo, as consultas de acompanhamento remotas, a fim de garantir o seu direito à vida e saúde.

Palavras-chave: Infecções por Coronavírus; Mães; Nascimento Prematuro; Pessoal de Saúde; Serviços de Saúde Materno-Infantil.

\section{Abstract}

Objective: The study aimed at understanding the repercussions of the Covid-19 pandemic in the care of premature infants, from the perspective of mothers and health professionals. Method: Semi-structured interviews were conducted in the months of June and July 2020, over the telephone, with 14 mothers and four health professionals from the follow-up service of a public maternity hospital in Paraíba, Brazil. Results: From the inductive thematic analysis, the impacts of the pandemic on the care of premature infants were as follows: overload and distancing of health service professionals, temporary deactivation of the Kangaroo mother unit, discontinuity of care for the premature infant, maternal fear of exposing the child to Covid-19 and low socioeconomic status. Coping strategies for the care of infants during the pandemic were listed, such as: greater spacing between consultations, phone follow-up and compliance with biosafety measures. Conclusion and implications for the practice: The pandemic required adaptations in care, which make new forms of care necessary for these children, such as remote follow-up consultations, in order to guarantee their right to life and health.

Keywords: Coronavirus Infections; Health Personnel; Premature Birth; Mothers; Maternal-Child Health Services.

\section{Resumen}

Objetivo: El estudio tuvo como objetivo comprender las repercusiones de la pandemia de Covid-19 en la atención de bebés prematuros, desde la perspectiva de las madres y los profesionales de la salud. Método: Se realizaron entrevistas semiestructuradas en los meses de junio y julio de 2020, por medio de llamadas telefónicas, a 14 madres y cuatro profesionales de la salud del servicio de seguimiento de una maternidad pública en Paraíba, Brasil. Resultados: A partir del análisis temático inductivo, los efectos de la pandemia en la atención de bebés prematuros fueron los siguientes: sobrecarga y distanciamiento de profesionales de los servicios de salud, inhabilitación temporal de la unidad Madre Canguro, discontinuidad de la atención al bebé prematuro, miedo materno a exponer al niño al Covid-19 y nivel socioeconómico bajo. Se enumeraron estrategias de afrontamiento para la atención infantil durante la pandemia, tales como: mayor intervalo entre consultas, seguimiento telefónico y cumplimiento de medidas de bioseguridad. Conclusión e implicaciones para la práctica: La pandemia requirió adaptaciones en la atención, que hacen necesarias nuevas formas de atención para estos niños, como las consultas de monitoreo remoto, para garantizar su derecho a la vida y a la salud.

Palabras clave: Infecciones por Coronavirus; Madres; Nacimiento Prematuro; Personal Sanitario; Servicios de Salud Materno-Infantil. 


\section{INTRODUÇÃO}

A Organização Mundial da Saúde (OMS) declarou, em 30 de janeiro de 2020, emergência internacional de saúde pública, em consequência da rápida disseminação da Corona Virus Disease (Covid-19), ocasionada pelo novo coronavírus (SARS-CoV-2), que surgiu em dezembro de 2019, na cidade de Wuhan, na China ${ }^{1}$. Com o aumento significativo das infecções por coronavírus em todo o mundo, em 11 de março de 2020, a Covid-19 foi caracterizada como uma pandemia ${ }^{2}$. Assim, a OMS recomendou que os países adotassem medidas de saúde pública, como a quarentena, o isolamento e o distanciamento social, além do funcionamento apenas de serviços essenciais, para reduzir a transmissão do vírus e/ou impedir o surgimento da doença em novos locais ${ }^{3}$.

Com relação ao funcionamento de serviços de saúde, o Ministério da Saúde (MS) brasileiro recomendou, no início de 2020, que as consultas de follow-up, ou seja, consultas para o acompanhamento do crescimento e desenvolvimento infantil, realizadas por uma equipe multiprofissional em ambulatórios de maternidades, fossem temporariamente suspensas, ficando, assim, sob responsabilidade das equipes de Atenção Primária à Saúde (APS) ${ }^{4}$.

Entretanto, estudos realizados em Unidades de Saúde da Família (USF) da Paraíba e do Piauí, constataram fragilidades na vigilância do desenvolvimento infantil, nesse ponto da Rede de Atenção à Saúde (RAS), com ações de cuidado realizadas de maneira pouco satisfatória ${ }^{5,6}$. Ademais, o mais preocupante é que, por ocasião da pandemia, as consultas de puericultura foram mantidas apenas para crianças de alto risco, resultando na descontinuidade do cuidado?.

A OMS destaca que a probabilidade de uma criança prematura sobreviver, crescer e se desenvolver plenamente depende, dentre outros fatores, do acesso oportuno aos atendimentos de saúde no nascimento e ao longo do seu crescimento e desenvolvimento ${ }^{8}$. Por isso, é fundamental o monitoramento da saúde da criança prematura bem como a existência de um sistema de saúde articulado e coordenado ${ }^{8}$.

No tocante à criança que nasceu prematura, diante da infecção pelo vírus SARS-CoV-2, a preocupação com ela é maior, devido à fragilidade do sistema imunológico, ainda imaturo, o que exige atenção prioritária, uma vez que a mesma poderá desenvolver formas graves da doença, requerendo suporte absoluto do sistema de saúde ${ }^{9}$.

A pandemia da Covid-19 trouxe inúmeras repercussões para a assistência prestada às crianças nascidas prematuras, como observado em estudos nacionais e internacionais que identificaram interrupção no acompanhamento de lactentes prematuros após a alta hospitalar, devido à paralização momentânea dos serviços de seguimento, com consequência para a descontinuidade da atenção à saúde prestada à essa população ${ }^{10,11}$.

Considerando a vulnerabilidade dos lactentes prematuros e a suspensão temporária das consultas de follow-up diante da pandemia assim como as medidas de isolamento tomadas que dificultaram o acompanhamento desse grupo no estado da Paraíba, o estudo se justifica pela relevância em refletir acerca do acompanhamento infantil frente à pandemia da Covid-19, tendo em vista a necessidade de cuidados contínuos à criança, especialmente, a prematura, com atenção mais vigilante no primeiro ano de vida.

Portanto, buscar compreender as repercussões da pandemia da Covid-19 no cuidado de lactentes prematuros na perspectiva de mães e profissionais de saúde é de fundamental relevância para a continuidade da assistência integral a esse grupo. Assim, o presente estudo tem como objetivo compreender as repercussões da pandemia de Covid-19 no cuidado de lactentes prematuros, na perspectiva de mães e profissionais de saúde.

\section{MÉTODO}

Trata-se de uma pesquisa descritivo-exploratória, com abordagem qualitativa, realizada em um ambulatório de followup, de egressos do método mãe canguru, de uma maternidade pública de um município da Paraíba, Brasil. Participaram do estudo 14 mães de lactentes que nasceram prematuros e quatro profissionais de saúde, que acompanhavam as crianças no referido ambulatório.

Adotaram-se como critérios de inclusão para as mães: ser mãe de lactentes egressos da unidade mãe canguru da referida maternidade, cujas crianças tiveram o acompanhamento de follow-up interrompido ou restrito devido à pandemia da Covid-19; ser maior de 18 anos e dispor de telefone celular. Para os profissionais de saúde, foi adotado o seguinte critério: realizar acompanhamento do crescimento e desenvolvimento dos lactentes prematuros no serviço de follow-up, antes da pandemia de Covid-19, por acreditarmos que esses teriam uma melhor visão do impacto da interrupção da consulta para algumas crianças.

Foram realizadas 56 ligações telefônicas para as mães de lactentes egressos, destas 13 não atenderam aos critérios de inclusão, cinco recusaram participar do estudo e não foi possível estabelecer contato com 24, assim, fizeram parte da pesquisa 14 mães de lactentes nascidos prematuros.

Este estudo contou com quatro entrevistadores com experiência em coleta de dados qualitativa. Esses foram previamente treinados pela pesquisadora principal, por meio de reuniões remotas. $\mathrm{O}$ teste piloto efetuado por todos os entrevistadores teve como propósito verificar a coerência e a compreensão das questões norteadoras, sendo validado por docente com expertise na temática e incluído nas entrevistas.

A coleta de dados ocorreu nos meses de junho e julho de 2020, utilizando a técnica de entrevista semiestruturada, audiogravada em mídia eletrônica, por meio de ligação telefônica, tendo em vista o isolamento social durante a pandemia da Covid-19. As mães foram recrutadas por conveniência, a partir do número de contato telefônico disponível no prontuário das crianças cadastradas no serviço de follow-up.

Inicialmente, as pesquisadoras entraram em contato com os participantes do estudo, por ligação telefônica, a fim de ter uma primeira aproximação com esses, apresentar os objetivos e convidá-los para participar da pesquisa. O agendamento das entrevistas com as mães ocorreu conforme disponibilidade e 
anuência, de modo que, em caso de recusa, contatava-se a seguinte de uma lista. Quanto aos profissionais de saúde, o recrutamento foi feito a partir de uma lista fornecida pelo gestor do referido serviço de saúde, contendo os respectivos números telefônicos.

O roteiro de entrevista continha duas partes, a primeira com informações sociodemográficas e a segunda com questões norteadoras. As entrevistas com as mães foram guiadas pelas seguintes perguntas: Como está ocorrendo o acompanhamento do seu filho na pandemia de Covid-19? Você percebeu alguma mudança na assistência? Se percebeu, o que você acha dessas mudanças em relação à assistência prestada ao seu filho? Já para os profissionais de saúde, as perguntas foram: Como está ocorrendo o acompanhamento do crescimento e desenvolvimento das crianças que nasceram prematuras na pandemia de Covid-19? Qual a sua percepção acerca das mudanças ocorridas na assistência infantil neste serviço, devido à Covid-19? As entrevistas duraram em média 20 minutos e o material empírico foi transcrito na íntegra, contudo, não foi realizada a devolutiva aos participantes da pesquisa.

O estudo buscou abranger todos os atores envolvidos no acompanhamento do crescimento e desenvolvimento dessas crianças. Para o encerramento da coleta das falas das mães, utilizou-se o critério de suficiência, quando foi possível refletir sobre as múltiplas dimensões do objeto de estudo ${ }^{12}$ o que totalizou 14 entrevistas. Vale salientar que, dos cinco profissionais atuantes no ambulatório de follow-up, apenas um não participou do estudo, tendo em vista que o mesmo ingressou no serviço durante a pandemia, quando as consultas de algumas crianças já tinham sido interrompidas.

Para a análise dos dados empíricos, utilizou-se a técnica de análise temática indutiva ${ }^{13}$, a qual é estruturada em seis fases: Familiarização com o tema, a qual exige leitura prévia e ativa do material empírico; Geração de códigos iniciais, para identificação de conjuntos semelhantes; Busca por temas, no intuito de selecionar diferentes códigos em temas potenciais; Revisão dos temas, a qual ocorre por meio da leitura de todos os extratos de dados que compõem cada tema e visualização da relação entre eles; Definição dos temas, para identificação dos mesmos e Produção textual final, com tratamento e interpretação dos resultados à luz da literatura pertinente ao tema.

O estudo vinculado a um projeto financiado pela Fundação de Apoio à Pesquisa do Estado da Paraíba (FAPESQ), atendeu aos preceitos éticos da Resolução 466/12 do Conselho Nacional de Saúde (CNS), com aprovação pelo Comitê de Ética em Pesquisa do Centro de Ciências da Saúde da Universidade Federal da Paraíba (UFPB), sob CAAE: 31353220.3.0000.5188.

A todos os participantes do estudo foi apresentado o Termo de Consentimento Livre e Esclarecido bem como a garantia do anonimato, por meio de codificação das falas com a letra "M", referente à Mãe, e "P" aos profissionais, ambas seguidas da numeração correspondente à ordem da entrevista, a saber: M1, M2 [...]; P1, P2 [...]. Destaca-se que cada anuência foi gravada verbalmente, após leitura do TCLE, antes de iniciadas as entrevistas por telefone.
Ressalta-se que foram utilizados neste estudo os critérios estabelecidos no Consolidated criteria for Reporting Qualitative Research (COREQ), como ferramenta de apoio em relação aos métodos de estudos qualitativos ${ }^{14}$.

\section{RESULTADOS}

Dos 18 participantes do estudo, 14 eram mães de lactentes que nasceram prematuros, e quatro eram profissionais de saúde que atuavam no follow-up. As mães tinham entre 23 e 38 anos, dentre elas, três tinham menos de oito anos de estudo, e 11 tinham oito anos ou mais. Quanto à renda familiar, nove recebiam até um salário mínimo, e cinco mais de um salário mínimo. Ademais, nove viviam em união estável, quatro eram solteiras e uma viúva.

Em relação aos profissionais de saúde, todos eram do sexo feminino, sendo uma enfermeira, uma fonoaudióloga, uma técnica de enfermagem e uma médica, com idades entre 38 e 57 anos. No tocante ao estado civil, três eram casadas e uma divorciada. Quanto ao tempo de atuação na assistência materno-infantil, duas tinham menos de 10 anos, e duas possuíam mais de 20 anos.

A partir da análise do material empírico, foram elaborados dois temas:Tema I: Repercussões da pandemia da Covid-19 na assistência ao lactente nascido prematuro; Tema II: Estratégias de enfrentamento para organização da atenção ambulatorial ao lactente nascido prematuro na pandemia da Covid-19, conforme a Figura 1.

\section{Tema I: Repercussões da pandemia da Covid-19 na assistência ao lactente nascido prematuro}

O contexto pandêmico repercutiu na assistência ofertada aos lactentes nascidos prematuros, nos diferentes pontos da RAS. Na atenção terciária, desvela-se que, com a pandemia, devido ao afastamento de alguns profissionais de saúde considerados grupo de risco, houve sobrecarga dos que continuaram realizando consultas no follow-up, apesar da redução do quantitativo de crianças para o atendimento:

Muitas vezes, eu fazia a busca ativa, ligava, procurava saber porque ela (a criança) não veio, enfim, fazia esse serviço, mas, como nossa demanda aumentou muito, eu termino não tendo tempo para isso. Nesse período de pandemia, parece que meu trabalho aumentou. Sem paciente, porém ficou mais puxado $(\mathrm{P} 1)$.

O grande problema da gente são os profissionais que se afastaram. No meu caso, uma colega que trabalhava comigo teve bebê e se afastou antes do parto e agora continua com a licença, e eu tenho que estar cobrindo a parte dela também (P2).

Ademais, outra repercussão da pandemia da Covid-19 na assistência aos prematuros foi a desativação temporária da Unidade de Cuidados Intermediários Canguru (UCINCa), setor primordial para a sobrevivência dessas crianças, aumentando 

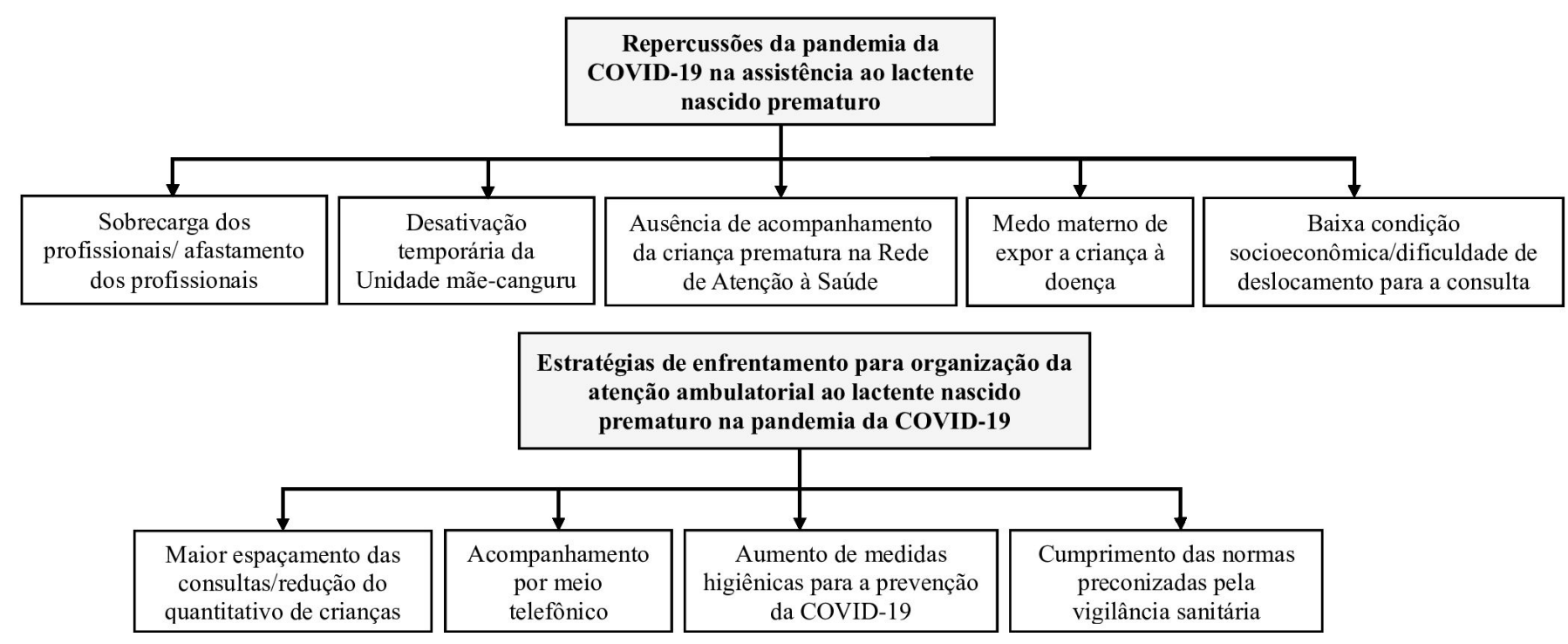

Figura 1. Mapa temático final, contendo os dois temas principais. João Pessoa, Paraíba, Brasil, 2020. Fonte: Dados da pesquisa.

ainda mais a vulnerabilidade dessas e a necessidade de uma assistência integral e contínua.

No momento da pandemia, está um pouco diferente, porque o serviço do método canguru foi desativado e os pacientes têm alta direto da UTI (Unidade de Terapia Intensiva) para casa (P2).

Por sua vez, quando questionadas sobre o acompanhamento do prematuro em outros pontos da RAS, como APS, as mães evidenciam que o atendimento à criança prematura também foi reduzido ou até mesmo interrompido.

Aí agora parou tudo, nem na Instituição X (instituição que atende a pessoa com deficiência) nem lá está tendo mais nada. Não, o postinho (USF) daqui também não está atendendo, assim consulta, só está atendendo urgência ou alguma receita (M17).

O postinho (USF) só está atendendo urgência. Só se tiver com caso de Covid-19. Até as vacinas lá estavam atrasadas, não queriam aplicar, aí eu fui, falei lá, aí aplicaram as vacinas dela e colocaram em dia (M7).

Observa-se que, além das modificações ocorridas nos serviços de atendimento à saúde durante a pandemia, houve absenteísmo de algumas crianças que deveriam continuar em atendimento, pois o medo materno de expor a criança ao risco de contrair a doença e a condição financeira familiar precária também repercutiram na assistência no follow-up, conforme extratos das falas de mães e profissionais de saúde.

Nós não estamos mais sendo acompanhados, porque ficamos com medo de viajar, ficamos com bastante medo, aí falamos com a médica, que ligou para marcar, mas não quisemos marcar porque ficamos com medo, porque a bebê não pode usar máscara e nós podemos, mas eles não, aí corre o risco do bebê pegar (M2).

Ela (profissional) disse que, quando melhorasse um pouquinho, retornasse, mas aí foi piorando a situação, aí eu não fui mais não, fiquei com medo... eu não vou mentir! (M1).

[...] Além disso, as mães têm muito medo de sair de casa, pegar o transporte público e até chegar ao hospital (P2).

As mães não têm condições financeiras, elas dão valor ao serviço, querem vir, mas não podem, não tem como, aí ficam faltando, atrasando. Isso prejudica realmente 0 acompanhamento da criança (P1).

\section{Tema II: Estratégias de enfrentamento para organização da atenção ambulatorial ao lactente nascido prematuro na pandemia da Covid-19}

O surgimento da Covid-19 exigiu dos serviços de saúde e das famílias estratégias de enfrentamento capazes de reduzir os impactos da pandemia na saúde do prematuro. Nessa perspectiva, o serviço de follow-up, como forma de evitar o contágio, aderiu a várias medidas, dentre elas, a redução do quantitativo de crianças atendidas e o aumento do espaçamento entre as consultas de acompanhamento daquelas que não poderiam deixar de serem atendidas.

Antes eles iam com 8, 15 dias (consulta), quando era normal, mas agora, com essa pandemia, está demorando mais (P3).

Eles estão fazendo em uma quantidade menor de pacientes, estão tomando todas as precauções, está entrando de um em um, e é isso (M21). 
A questão de quantidade (atendimento) por dia também para evitar aglomeração, a gente está mantendo uma média de 7 a 8 pacientes por dia, antes era uma média de 12 a 15. A gente diminuiu por conta da aglomeração. Estamos solicitando o uso de máscaras até para quem está aguardando na sala de espera, e as cadeiras estão marcadas com cadeiras disponíveis e não disponíveis para a gente manter o distanciamento (P4).

Frente às limitações do atendimento presencial ao prematuro no ambulatório de follow-up durante a pandemia, para atender às necessidades da criança e de seus familiares, foi implementado pelo serviço o atendimento telefônico, como uma forma de não causar ruptura total no acompanhamento dessas crianças.

Ele não está tendo atendimento aí (follow-up), pessoalmente, mas está tendo atendimento pelo celular, porque qualquer dúvida minha, qualquer coisinha que ele sente, eu ligo, e aí a médica atende ele, me passa tudo pelo celular [...]A médica tira todas as minhas dúvidas quando eu ligo para ela, me explica tudo, parece que eu estou pessoalmente com ela. Em relação a isso, continua para mim a mesma coisa (M9).

Nós sempre mantemos contato pelo WhatsApp, por telefone e nós sempre estamos tendo notícia dos bebês, sabe? Quando elas não vêm para consulta, elas mandam alguma pergunta, e a gente está sempre respondendo e tentando não perder esse vínculo, que é muito importante (P2).

Quando a mãe está com alguma dúvida, está com pouco leite, aí eu peço para fazer um vídeo para ver como está sugando, se for o caso, oriento uma posição melhor. Às vezes, elas mandam um vídeo, e você consegue fazer uma comparação com o vídeo anterior que ela mandou. Assim, você vai fazendo o acompanhamento do desenvolvimento daquela criança. A gente não quer que elas se afastem (P4).

Apesar das adversidades oriundas do cenário pandêmico, houve mudança na organização da atenção ambulatorial ao lactente prematuro, com mais precaução no que diz respeito às medidas higiênicas para a prevenção do contágio da Covid-19, tanto por parte dos profissionais de saúde quanto das mães.

Os pais estão tendo que ficar mais tempo dentro de casa e, consequentemente, estão dando mais atenção à criança também. Hoje em dia, já são mais receosos, eles estão cuidando mais da criança. Eu vejo que está tendo uma higiene melhor com a criança e com a própria mãe, estão se cuidando mais (P4)

Minha rotina modificou em relação aos EPIs (Equipamentos de Proteção Individual), porque antes não usávamos tanto quanto hoje (P4).
A gente está tendo todos os cuidados como preconiza a vigilância [...] se nós tínhamos antes cuidados, hoje já fica de uma forma redobrada; o cuidado fica maior ainda no sentido de sempre estar paramentado, sempre que for fazer algum procedimento, lavar as mãos e usar os EPIs; nunca deixar de usar, principalmente, isso (P1).

[...] eu passei a dobrar mais os cuidados em relação a ela. Um exemplo: antes eu saía para a rua, para levar ela para vacinar, quando a gente voltava, se não tivesse sujinha, eu não dava banho, mas hoje não, quando vai se vacinar, quando volta, a gente dá banho. A gente também tem uma mascarazinha pequenininha que a gente fez aqui em casa para ela. Do mesmo jeito, é no meu caso, tiro toda minha roupa, coloco para lavar e tomo banho (M6).

\section{DISCUSSÃO}

A crise decorrente da pandemia da Covid-19 provocou inúmeras transformações, tanto no ambiente doméstico, como nos serviços de saúde que prestam atendimento aos lactentes nascidos prematuros. Uma das repercussões na assistência ofertada pelos serviços da atenção terciária foi a sobrecarga dos profissionais atuantes no follow-up, devido à redução do quantitativo de pessoal, pois alguns que lá trabalham foram afastados, por serem considerados grupos de risco para a doença. Desse modo, tanto os profissionais de saúde quanto as famílias de lactentes prematuros precisaram se reinventar para que o cuidado à criança não fosse prejudicado.

Diversas mudanças nos processos de trabalho e na assistência em saúde foram necessárias, como: instituição de novos protocolos de atendimento, utilização de EPIs, antes não utilizados em vários contextos; suspensão de alguns atendimentos; afastamento ou realocação de trabalhadores considerados grupos de risco, entre outros aspectos que dificultam o cuidado em saúde ${ }^{15,16} \mathrm{e}$ que causam sobrecarga física e emocional nos profissionais.

Nessa diretiva, os extratos das falas revelam que o cenário pandêmico modificou consideravelmente a assistência às crianças e o contexto de trabalho dos profissionais da saúde. No entanto, essas alterações foram necessárias, tendo em vista que o processo de trabalho em saúde é dinâmico, transformando-se a partir das ações dos trabalhadores que, por meio do saber operante e de modelos tecnológicos, modificam seus contextos laborais, a fim de satisfazer finalidades sociais ${ }^{17}$.

Assim, com a pandemia, surgiu o estresse decorrente do aumento das demandas de trabalho dos profissionais de saúde e os efeitos dessa situação, como o crescente medo e insegurança em relação ao alto risco de contágio da doença, o que acarreta afastamentos dos serviços e, por sua vez, descontinuidade da assistência prestada ${ }^{18,19}$. Isso condiz com os discursos dos participantes do presente estudo, que relatam aumento da carga de trabalho em virtude dos desfalques no quadro de funcionários.

É importante destacar que, apesar de a Covid-19 ter acometido poucos Recém-nascidos (RN), a doença tem gerado 
importantes mudanças no modelo de atenção humanizada ao Recém-Nascido Pré-termo (RNPT) e/ou de baixo peso, como evidenciado nos resultados deste estudo, que destacam a interrupção temporária da segunda etapa do Método Canguru (MC). Essa descontinuidade do método é contrária às atuais diretrizes do MS, que recomendam que as UCINCas não devem ser fechadas nem restritas durante a pandemia, uma vez que a finalidade do MC é reduzir os índices de morbimortalidade infantili ${ }^{20}$.

A estratégia mãe canguru deve ocorrer em três etapas: a primeira desenvolve-se durante a internação do recém-nascido prematuro e/ou de baixo peso na Unidade Intensiva Neonatal ou na Unidade de Cuidado Intermediário Neonatal Convencional; na segunda, o bebê permanece, de maneira contínua, com sua mãe, na posição canguru, na UCINCa, e a terceira etapa é domiciliar, com seguimento compartilhado entre a unidade ambulatorial da maternidade onde o bebê nasceu e a Unidade Básica de Saúde, até o RN atingir o peso de $2.500 \mathrm{~g}^{21}$.

Portanto, a interrupção do MC pode se tornar um fator de risco para os RNPT e/ou de baixo peso, pois a ausência do cuidado proposto pelo método e a falta de uma assistência integral e qualificada podem ocasionar sérios danos e deficiências a essas crianças ${ }^{22}$.

Na presente pesquisa, o seguimento aos lactentes nascidos prematuros foi interrompido causando insegurança para família na prestação de cuidados. Tal realidade poderá repercutir no desenvolvimento neuropsicomotor da criança, bem como na sua qualidade de vida, visto essa interrupção dificulta o acompanhamento da saúde da criança, com repercussões futuras, tendo em vista que os primeiros anos de vida da criança são primordiais para o seu pleno desenvolvimento. Isso acontece devido à plasticidade cerebral, quando novas experiências vividas pelos bebês favorecem a formação de outras conexões cerebrais. Assim, esse período corresponde a melhor oportunidade para favorecer o desenvolvimento cognitivo, físico, da saúde e educação, e para moldar positivamente o futuro das crianças ${ }^{23,24}$.

Os extratos das falas dos participantes do estudo também revelam que, para garantir o seguimento ao prematuro, a assistência ofertada precisou ser modificada com medidas de prevenção de contágio, exigindo dos profissionais estratégias capazes de reduzir os impactos da pandemia na saúde do prematuro, como o aumento do espaçamento entre as consultas e a redução da quantidade de atendimentos ambulatoriais. Entretanto, essa nova organização do trabalho para o atendimento e assistência pode contribuir para perda significativa do vínculo ou mesmo o rompimento da relação profissional-paciente ${ }^{25}$.

Assim, para o enfrentamento da pandemia, são necessárias readaptações na rotina dos profissionais e dos serviços de saúde, com incorporação de estratégias, para que o serviço funcione, incluindo novas formas de cuidado à distância, com o uso de tecnologias de informação e comunicação, como mídia social e telefone celular, para a realização de teleconsulta ${ }^{26}$.

Essa é uma estratégia viável para garantir a assistência ao lactente prematuro e atender às necessidades da criança e de seus familiares, uma vez que o suporte telefônico no cenário pandêmico pode ser uma alternativa viável para evitar a descontinuidade do acompanhamento dessas crianças. Todavia, é preciso discernir as situações que exigem contato com o binômio mãe-filho, sendo dificilmente substituídos pela consulta online ${ }^{27}$.

Ademais, o acompanhamento por meio telefônico favorece a apreensão de necessidades de saúde e esclarecimento de dúvidas das mães acerca dos cuidados básicos e cotidianos à criança ${ }^{28}$, conforme evidenciou uma das participantes do estudo, ao mencionar que o acompanhamento e a continuidade do cuidado por telefone foram capazes de solucionar problemas de saúde dos seus filhos.

Outra repercussão do cenário pandêmico, apontada neste estudo, foi o comprometimento das consultas às crianças nas USF, devido à redução ou até mesmo à interrupção do acompanhamento à criança na puericultura. Estudo realizado em ambulatórios pediátricos na Itália evidenciou que houve $98,2 \%$ de redução das consultas durante o período pandêmico ${ }^{29}$.

Essa interrupção é preocupante, tendo em vista que o seguimento à saúde da criança possibilita a detecção precoce de possíveis agravos e déficits na infância, a partir da vigilância do desenvolvimento infantil. Essa compõe uma tecnologia leve em saúde, que permite intervir nos agravos, em tempo oportuno, minimizando os riscos e prevenindo a ocorrência da morbimortalidade ${ }^{5}$.

Consequente a esse cenário, a cobertura vacinal de rotina também sofreu impacto nos serviços de saúde, com redução expressiva pela oferta e procura das vacinas, igualmente constatado em pesquisas nos Estados Unidos, que apontam um declínio na imunização de rotina ${ }^{30,31}$. Isso coaduna com os achados do presente estudo, visto que os relatos desvelam dificuldades para atualizar o calendário vacinal da criança.

É pertinente ressaltar que, em situação de pandemia, a interrupção de cuidados básicos, a exemplo da vacinação, constitui um risco potencial para crises ainda mais graves na saúde, com surtos de outras doenças. Sendo assim, a OMS juntamente com o Fundo das Nações Unidas para a Infância (UNICEF) e as Sociedades Brasileiras de Pediatria e de Imunização recomendam a reorganização dos serviços para continuar com o funcionamento das salas de vacinas e manter a rotina com segurança, devendo priorizar os menores de cinco anos, gestantes e grupos de risco, por meio de atendimento no serviço ou extramuro, desde que cumprindo as medidas de prevenção para o novo coronavírus ${ }^{3,32}$.

Apesar de algumas fragilidades na atuação das equipes da Estratégia Saúde da Família, esse é o modelo mais adequado e eficiente para o apoio às populações em situação de isolamento social, pois é preciso manter o contato e o vínculo das pessoas com os profissionais responsáveis pelo cuidado à saúde ${ }^{26}$.

No tocante à assistência ao lactente que nasceu prematuro, os achados deste estudo apontaram influência de fatores como o medo das mães em expor os filhos ao risco de contágio pelo vírus SARS-CoV-2, sobretudo, no deslocamento para os serviços de saúde, visto a necessidade de utilizar transporte público, 
situação que resultou na descontinuidade da assistência à criança. Fato semelhante foi observado em pesquisa realizada com familiares de crianças italianas com necessidades especiais, a qual evidenciou que, devido ao medo da infecção pelo novo coronavírus, mesmo em casos de adoecimento, evitavam levá-las ao serviço de saúde, o que resultou em agravamento do quadro clínico, em metade da amostra, e no óbito de quatro crianças ${ }^{33}$.

Ainda com relação às estratégias de enfrentamento para a assistência da criança nascida prematura, destaca-se a intensificação de medidas de higiene e uso de EPIs pelo pessoal de saúde. Estudos nacionais revelam que os profissionais se tornaram mais conscientes com medidas de higiene das mãos e desinfecção com álcool a $70 \%$ e com uso de equipamentos de proteção individual ${ }^{6,34}$.

A mudança nos cuidados não foi exclusiva dos profissionais de saúde, pois as famílias e a comunidade tiveram que adotar novos hábitos de higiene e isolamento social. Dentre as medidas citadas pelas mães, que passaram a ser mais cautelosas com seus filhos, estão: aumento da higienização das mãos com água e sabão, álcool a $70 \%$, além de banho ao retornar para casa. Os resultados são condizentes com pesquisa internacional realizada na Jordânia, na qual foi evidenciado que as famílias evitavam sair de seus domicílios, higienizavam frequentemente suas mãos e as das crianças e também costumavam limpar e desinfetar as superfícies com que a criança entra em contato, como medidas preventivas ao novo coronavírus ${ }^{35}$.

Cabe ressaltar que essas medidas de higiene e isolamento social muitas vezes não são possíveis de serem executadas pelas comunidades mais vulneráveis, pois muitas famílias dispõem de acesso precário ao saneamento básico e à água potável bem como não conseguem manter o isolamento diante do contexto familiar e social ${ }^{36}$ e também não têm condições financeiras para comprar álcool em gel $70 \%$. Essa realidade exige um olhar diferenciado e ampliado dos profissionais de saúde acerca do contexto em que a criança e família vivem, para, assim, planejarem estratégias de enfrentamento à Covid-19 e continuidade do cuidado.

Diante do exposto, destaca-se a importância da utilização de estratégias para adequar os serviços e ações de saúde às mudanças dos contextos sociais em que as crianças e suas famílias estão inseridas, para, assim, garantir a continuidade do vínculo com os profissionais e promover uma assistência integral e longitudinal a esse grupo.

\section{CONCLUSÃO E IMPLICAÇÕES PARA A PRÁTICA}

As repercussões da pandemia da Covid-19 no cuidado de lactentes prematuros perpassam por sobrecarga profissional, limitação no acompanhamento do prematuro na RAS, medo materno de expor a criança à doença e diminuição das condições socioeconômicas das famílias. Quanto às estratégias de enfrentamento para o cuidado, diante da pandemia no setor de follow-up, observou-se aumento do espaçamento das consultas, acompanhamento da saúde da criança por telefone e ampliação das medidas de higiene e precauções.

O novo contexto, desafiador e repleto de incertezas, impôs aos familiares e profissionais adaptações para garantia de um cuidado qualificado e efetivo ao lactente prematuro. Ademais, a necessidade de reorganização dos serviços e do processo de trabalho dos profissionais de saúde exigiu uma atuação mais dinâmica diante das demandas da criança prematura em tempos de pandemia.

Os achados desse estudo trazem como implicações para a prática profissional o direcionamento para estratégias de cuidado e acompanhamento às crianças nascidas prematuras que se adequem ao contexto da pandemia, garantindo um cuidado integral e longitudinal. Assim, tornam-se necessárias novas formas de assistência para essas crianças, como as consultas de acompanhamento remotas, a fim de garantir o seu direito à vida e à saúde.

Como limitação do estudo, destaca-se o fato de as entrevistas terem acontecido por meio telefônico, o que pode ter dificultado a interação entre os pesquisadores e os participantes da pesquisa, como também, pode ter contribuído para reduzir o tempo da fala, uma vez que a entrevista presencial facilita o diálogo. Além disso, o formato remoto de entrevista pode ter fragilizado a apreensão das repercussões da pandemia na família, visto que a presença do pesquisador no ambiente familiar poderia favorecer uma compreensão mais profunda das vulnerabilidades das crianças que nasceram prematuras e de suas famílias.

\section{FINANCIAMENTO}

Fundação de Apoio à Pesquisa do Estado da Paraíba (FAPESQ) para o projeto de pesquisa intitulado "Assistência materno infantil frente à pandemia de Covid-19: uma proposta de cuidado a distância", coordenado pela pesquisadora Altamira Pereira da Silva Reichert. Número do processo: 40643.608.30727.15042020.

\section{CONTRIBUIÇÕES DOS AUTORES}

Desenho do estudo. Altamira Pereira da Silva Reichert. Nathanielly Cristina Carvalho de Brito Santos.

Coleta ou produção dos dados. Anna Tereza Alves Guedes. Anniely Rodrigues Soares. Paloma Karen Holanda Brito. Layla Caroline Lino da Silva.

Análise de dados. Altamira Pereira da Silva Reichert. Anna Tereza Alves Guedes. Anniely Rodrigues Soares. Nathanielly Cristina Carvalho de Brito Santos.

Interpretação dos resultados. Altamira Pereira da Silva Reichert. Anna Tereza Alves Guedes. Anniely Rodrigues Soares. Paloma Karen Holanda Brito. Iolanda Carlli da Silva Bezerra. Layla Caroline Lino da Silva. Tayanne Kiev Carvalho Dias. Nathanielly Cristina Carvalho de Brito Santos

Redação e revisão crítica do manuscrito. Altamira Pereira da Silva Reichert. Anna Tereza Alves Guedes. Anniely Rodrigues Soares. Paloma Karen Holanda Brito. Iolanda Carlli da Silva 
Bezerra. Layla Caroline Lino da Silva. Tayanne Kiev Carvalho Dias. Nathanielly Cristina Carvalho de Brito Santos.

Aprovação da versão final do artigo. Altamira Pereira da Silva Reichert. Anna Tereza Alves Guedes. Anniely Rodrigues Soares. Paloma Karen Holanda Brito. Iolanda Carlli da Silva Bezerra. Layla Caroline Lino da Silva. Tayanne Kiev Carvalho Dias. Nathanielly Cristina Carvalho de Brito Santos.

Responsabilidade por todos os aspectos do conteúdo e a integridade do artigo publicado. Altamira Pereira da Silva Reichert. Anna Tereza Alves Guedes. Anniely Rodrigues Soares. Paloma Karen Holanda Brito. Iolanda Carlli da Silva Bezerra. Layla Caroline Lino da Silva. Tayanne Kiev Carvalho Dias. Nathanielly Cristina Carvalho de Brito Santos.

\section{EDITOR ASSOCIADO}

\author{
Eliane Tatsch Neves (D)
}

\section{EDITOR CIENTÍFICO}

Ivone Evangelista Cabral (D)

\section{REFERÊNCIAS}

1. World Health Organization. WHO Director-General's opening remarks at the media briefing on COVID-19. Geneva:WHO; 2020 [citado 2020 outubro 12]. Disponivel em: https://www.who.int/dg/speeches/detail/ who-director-general-s-opening-remarks-at-the-media-briefing-oncovid-19---28-september-2020

2. World Health Organization. Considerations for quarantine of individuals in the context of containment for coronavirus disease (COVID-19): interim guidance, 19 March 2020 [Internet]. Geneva:WHO; 2020 [citado 2020 outubro 12]. Disponível em: https://apps.who.int/iris/handle/10665/331497

3. World Health Organization. Routine immunization services during the COVID-19 pandemic [Internet]. Geneva:WHO; 2020 [citado 2020 outubro 12]. Disponível em: https://apps.who.int/iris/handle/10665/331925

4. Ministério da Saúde (BR). Nota técnica no 6/2020 - COCAM/CGCIVI/ DAPES/SAPS/MS. Atenção à Saúde do Recém-nascido no Contexto da Infecção do novo coronavírus (SARS-COV-2) [Internet]. Brasília: Ministério da Saúde;2020 [citado 2020 outubro 12]. Disponível em: http://docs.bvsalud. org/biblioref/2020/04/1087595/notatecnicaneonatal30mar2020covid-19. pdf

5. Vieira DS, Santos NCCB, Nascimento JA, Collet N, Toso BRGO, Reichert APS. A prática do enfermeiro na consulta de puericultura na Estratégia Saúde da Família. Texto Contexto Enferm. 2018;27(4):1-10. http://dx.doi.org/10.1590/0104-07072018004890017.

6. Oliveira FFS, Oliveira ASS, Lima LHO, Marques MB, Felipe GF, Sena IVO. Child care consultations held by nurses within the Family health strategy. Rev Rene. 2013; [citado 2020 outubro 12];14(4):694-703. Disponível em: http://www.periodicos.ufc.br/rene/article/view/3526

7. Paraná. Governo do Estado. Secretária de Saúde do Estado do Paraná. Coronavírus (COVID-19) - Nota orientativa no 29/2020 - Atendimento em puericultura. Curitiba: Secretária de Saúde do Estado do Paraná; 2020 [citado 2020 outubro 12]. Disponível em: https://www.saude. pr.gov.br/sites/default/arquivos_restritos/files/documento/2020-06/ no_29_atendimento_em_puericultura.pdf

8. World Health Organization. Survive and thrive: transforming care for every small and sick newborn [Internet]. Geneva: WHO; 2019 [citado 2020 outubro 12]. Disponível em: https://www.who.int/maternal_child adolescent/documents/care-small-sick-newborns-survive-thrive/en/

9. Wang L, ShiY, Xiao T, Fu J, Feng X, Mu D et al. Chinese expert consensus on the perinatal and neonatal management for the prevention and control of the 2019 novel coronavirus infection (First edition). Ann Transl Med. 2020;8(3):1-8. http://dx.doi.org/10.21037/atm.2020.02.20.
10. Silva RMM, Pancieri L, Zilly A, Spohr FA, Fonseca LMM, Mello DF. Follow-up care for premature children: the repercussions of the COVID-19 pandemic. Rev Lat Am Enfermagem. 2021;29:e3414. http://dx.doi. org/10.1590/1518-8345.4759.3414. PMid:33852686.

11. Li L, Li Z, Wan W, Li J, Zhang Y, Wang $C$ et al. Management of follow-up with preterm infants during the outbreak in China. Front Pediatr. 2021;9:637275. http://dx.doi.org/10.3389/fped.2021.637275. PMid:33996687.

12. Minayo MCS. Amostragem e saturação em pesquisa qualitativa: consensos e controvérsias. Rev Pesqui Qual (Online). [Internet]. 2017; [citado 2020 outubro 12];5 (7):1-12. Disponível em: https://editora.sepq. org.br/index.php/rpq/article/view/82/59

13. Braun V, Clarke V. Using thematic analysis in psicology. Qual Res Psychol. 2006;3(2):77-101. http://dx.doi.org/10.1191/1478088706qp063oa.

14. Tong A, Sainsbury P, Craig J. Consolidated criteria for reporting qualitative research (COREQ): a 32 -item checklist for interviews and focus groups. Int J Qual Health Care. 2007;19(6):349-57. http://dx.doi.org/10.1093/ intqhc/mzm042. PMid:17872937.

15. Adams JG, Walls RM. Supporting the health care workforce during the COVID-19 global epidemic. JAMA. 2020;323(15):1439-40. http://dx.doi. org/10.1001/jama.2020.3972. PMid:32163102.

16. Teixeira CFDS, Soares CM, Souza EA, Lisboa ES, Pinto ICDM, Andrade LRD et al. A saúde dos profissionais de saúde no enfrentamento da pandemia de Covid-19. Cien Saude Colet. 2020;25(9):3465-74. http:// dx.doi.org/10.1590/1413-81232020259.19562020. PMid:32876270.

17. Peduzzi M, Anselmi ML. O processo de trabalho de enfermagem: a cisão entre planejamento e execução do cuidado. Rev Bras Enferm. 2002;55(4):392-8. http://dx.doi.org/10.5935/0034-7167.20020086. PMid:12728804.

18. Almaghrabi RH, Alfaraidi HA, Al Hebshi WA, Albaadani MM. Healthcare workers experience in dealing with Coronavirus (COVID-19) pandemic. Saudi Med J. 2020;41(6):657-60. http://dx.doi.org/10.15537/ smj.2020.6.25101. PMid:32518935.

19. Góes FGB, Silva ACSS, Santos AST, Pereira-Ávila FMV, Silva LJ, Silva LF et al. Challenges faced by pediatric nursing workers in the face of the COVID-19 pandemic. Rev Lat Am Enfermagem. 2020;28:e3367. http://dx.doi.org/10.1590/1518-8345.4550.3367. PMid:32901774.

20. Ministério da Saúde (BR). Nota técnica no 6/2020 COCAM/CGCIVI/ DAPES/SAPS/MS - Atenção à Saúde do Recém-nascido no Contexto da Infecção do novo coronavírus (SARS-COV-2) [Internet]. Brasília: Ministério da Saúde; 2020 [citado 2020 outubro 12]. Disponível em: https://www.unasus.gov.br/especial/covid19/pdf/97

21. Ministério da Saúde (BR). Método Canguru: manual da terceira etapa do Método Canguru na Atenção Básica [Internet]. Brasília: Ministério da Saúde; 2018 [citado 2020 outubro 12]. Disponível em: http://bvsms. saude.gov.br/bvs/publicacoes/manual_terceira_etapa_metodo_canguru. pdf

22. Ministério da Saúde (BR). Atenção humanizada ao recém-nascido: Método Canguru: manual técnico [Internet]. Brasília: Ministério da Saúde;2017 [citado 2020 outubro 12]. Disponível em: http://bvsms.saude.gov.br/ bvs/publicacoes/atencao_humanizada_metodo_canguru_manual_3ed. pdf

23. Sparling J, Meunier K. Abecedarian: an early childhood education approach that has a rich history and a vibrant present. Int J Early Child. 2019;51(2):207-16. http://dx.doi.org/10.1007/s13158-019-00247-2.

24. Mushtaq A, Kazi F. The case for early child development programmes. Lancet Child Adolesc Health. 2019;3(12):851. http://dx.doi.org/10.1016/ S2352-4642(19)30298-6.

25. Sachett JAG. Adaptação para o atendimento profissional de saúde em tempos de COVID-19: contribuições da telessaúde para o "novo normal". J Health NPEPS. 2020;5(2):11-5. http://dx.doi.org/10.30681/252610104877.

26. Medina MG, Giovanella L, Bousquat A, Mendonça MHM, Aquino R, Comitê Gestor da Rede de Pesquisa em Atenção Primária à Saúde da Abrasco. Atenção primária à saúde em tempos de COVID-19: o que fazer? Cad Saude Publica. 2020;36(8):e00149720. http://dx.doi. org/10.1590/0102-311x00149720. PMid:32813791.

27. Sarti TD, Lazaríni WS, Fontenelle LF, Almeida APS. Qual o papel da Atenção Primária à Saúde diante da pandemia provocada pela 
COVID-19? Epidemiol Serv Saude. 2020;29(2):e2020166. http://dx.doi. org/10.5123/S1679-49742020000200024. PMid:32348404.

28. Silva RMM, Zilly A, Nonose ERS, Fonseca LMM, Mello DF. Care opportunities for premature infants: home visits and telephone support. Rev Lat Am Enfermagem. 2020;28:e3308. http://dx.doi.org/10.1590/15188345.3520.3308. PMid:32609266.

29. Bechini A, Garamella G, Giammarco B, Zanella B, Flori V, Bonanni $P$ et al. Paediatric activities and adherence to vaccinations during the COVID-19 epidemic period in Tuscany, Italy: a survey of paediatricians. J Prev Med Hyg. 2020;61(2):E125-9. http://dx.doi.org/10.15167/24214248/jpmh2020.61.2.1626. PMid:32802994.

30. Santoli JM, Lindley MC, DeSilva MB, Kharbanda EO, Daley MF, Galloway $L$ et al. Effects of the COVID-19 pandemic on routine pediatric vaccine ordering and administration - United States, 2020. MMWR Morb Mortal Wkly Rep. 2020;69(19):591-3. http://dx.doi.org/10.15585/mmwr. mm6919e2. PMid:32407298.

31. Vogt TM, Zhang F, Banks M, Black C, Arthur B, Kang Y et al. Provision of Pediatric Immunization Services During the COVID-19 Pandemic: an Assessment of Capacity Among Pediatric Immunization Providers Participating in the Vaccines for Children Program - United States,
May 2020. MMWR Surveill Summ. 2020;69(27):859-63. http://dx.doi. org/10.15585/mmwr.mm6927a2. PMid:32644980.

32. Sociedade Brasileira de Pediatria. Pandemia da Covid-19 - O que muda na rotina das Imunizações [Internet]. 2020. [citado 2021 maio 4]. Disponível em: https://portaldeboaspraticas.iff.fiocruz.br/biblioteca/ pandemia-da-covid-19-o-que-muda-na-rotina-das-imunizacoes/

33. Lazzerini M, Barbi E, Apicella A, Marchetti F, Cardinale F, Trobia G. Delayed access or provision of care in Italy resulting from fear of Covid-19. Lancet Child Adolesc Health. 2020;4(5):e10-1. http://dx.doi. org/10.1016/S2352-4642(20)30108-5. PMid:32278365.

34. Rodrigo JAP, Stelmatchuk AM, Lacerda MR, Galvão CM. Medidas de contenção à Covid-19 adotadas em serviço de transplante de medula óssea. Rev Bras Enferm. 2020;73(supl 2):e20200476. http://dx.doi. org/10.1590/0034-7167-2020-0476.

35. Abuhammad S. Parents' knowledge and atitude towards COVID-19 in children: a Jordanian study. Int J Clin Pract. 2021 fev;75(2):e13671. http://dx.doi.org/10.1111/ijcp.13671. PMid:32780560.

36. Souza MN. Desigualdade e seletividade social das medidas de contenção da Covid-19 na periferia de Curitiba. Guaju. 2020;6(1):131-46. http:// dx.doi.org/10.5380/guaju.v6i1.73654. 